\title{
Improved intuitionistic fuzzy composite relation and its application to medical diagnostic process
}

\author{
P. A. Ejegwa ${ }^{1}$ and B. O. Onasanya ${ }^{2}$ \\ ${ }^{1}$ Department of Mathematics, Statistics and Computer Science \\ University of Agriculture, P.M.B. 2373, Makurdi, Nigeria \\ e-mail: ocholohi@gmail.com \\ ${ }^{2}$ Department of Mathematics, Faculty of Science \\ University of Ibadan, Nigeria \\ e-mail: babtu2001@yahoo.com
}

Received: 18 December 2018

Revised: 24 March 2019

Accepted: 6 April 2019

\begin{abstract}
In this paper, we study the De et al.'s approach for the application of intuitionistic fuzzy relation (i.e., max-min-max composite relation), and improve the approach for better output. The validity of the improved intuitionistic fuzzy composite relation is carried out in comparison to De et al.'s approach using numerical experiments. It is shown that the improved intuitionistic fuzzy composite relation yields a better output. Finally, an application of the improved approach to medical diagnostic process is carried out using a hypothetical medical database. This improved intuitionistic fuzzy composite relation could be used as a sustainable approach in applying intuitionistic fuzzy sets to other real-life decision-making problems.
\end{abstract}

Keywords: Fuzzy set, Intuitionistic fuzzy set, Intuitionistic fuzzy relation, Intuitionistic fuzzy medical diagnosis.

2010 Mathematics Subject Classification: 20N20, 03E72.

\section{Introduction}

Fuzzy set theory proposed by Zadeh [31] has many applications because of its ability to cope with imprecision embedded in real-life decision-making problems. For a classical set $X$, a fuzzy 
set over $X$, or a fuzzy subset of $X$, is characterized by a membership function $\mu$ which associates values from the closed unit interval $I=[0,1]$ to members of $X$. Fuzzy sets are able to model vagueness. However, they cannot model uncertainty precisely because there is no means to attribute reliability information to the membership degrees [17]. The vast presence of uncertainty in day-to-day life necessitated researchers to develop some mathematical frameworks that can handle uncertainty more accurately than fuzzy sets.

Furthermore, out of the several mathematical frameworks which generalize fuzzy sets, the concept of intuitionistic fuzzy sets (IFSs) introduced by Atanassov [1] is interesting and resourceful. In fact, every fuzzy set is an IFS but the converse is not necessarily true [2]. The concept of IFS theory has been extensively studied, for details see [3, 4, 6, 13].

The theory of IFSs has been applied in different decision-making problems, viz., logic programming, medical diagnosis, electoral system, career determination, appointment procedures, pattern recognition, learning techniques, among others (see [5, 7, 8, 14, 18-23, 25-30] for details).

Albeit, in a way to extend the notion of fuzzy relations [15,24] and its application [16] to IFS setting, Burillo and Bustince [10] explored the concept of intuitionistic fuzzy relations (IFRs). More works on the structures of IFRs have been explicated in $[9,11]$. In a nutshell, IFRs are IFSs in a Cartesian product of universes [17]. The method of intuitionistic fuzzy medical diagnosis that involves intuitionistic fuzzy relations as defined in [9] was carried out by De et al. [14].

In this paper, we study the De et al.'s approach of medical diagnostic process using IFS theory and improved upon the approach for better output. The paper is organized by presenting some basic notions of IFSs in Section 2. In Section 3, we discuss the concept of IFRs comprises of intuitionistic fuzzy max-min-max composite relation, improved intuitionistic fuzzy composite relation and their numerical verifications. Section 4 expatiates the application of the improved intuitionistic fuzzy composite relation in medical diagnostic process using the medical database in [14]. Finally, Section 5 concludes the paper and provides direction for future studies.

\section{Some basic notions of intuitionistic fuzzy sets}

In this section, we recall some mathematical preliminaries of IFSs.

Definition 2.1. [31] Let $X$ be a nonempty set. A fuzzy set $A$ of $X$ is characterized by a membership function

$$
\mu_{A}: X \rightarrow[0,1]
$$

That is,

$$
\mu_{A}(x)= \begin{cases}1, & \text { if } x \in X \\ 0, & \text { if } x \notin X \\ (0,1) & \text { if } x \text { is partly in } X\end{cases}
$$

Alternatively, a fuzzy set $A$ of $X$ is an object having the form

$$
A=\left\{\left\langle x, \mu_{A}(x)\right\rangle \mid x \in X\right\} \text { or } A=\left\{\left\langle\frac{\mu_{A}(x)}{x}\right\rangle \mid x \in X\right\},
$$


where the function $\mu_{A}(x)$ defines the degree of membership of the element $x \in X$.

Definition 2.2. [1,2] Let a nonempty set $X$ be fixed. An IFS $A$ of $X$ is an object having the form

$$
A=\left\{\left\langle x, \mu_{A}(x), \nu_{A}(x)\right\rangle \mid x \in X\right\}
$$

or

$$
A=\left\{\left\langle\frac{\mu_{A}(x), \nu_{A}(x)}{x}\right\rangle \mid x \in X\right\}
$$

where the functions

$$
\mu_{A}(x): X \rightarrow[0,1] \text { and } \nu_{A}(x): X \rightarrow[0,1]
$$

define the degree of membership and the degree of non-membership, respectively of the element $x \in X$ to $A$, which is a subset of $X$, and for every $x \in X$,

$$
0 \leq \mu_{A}(x)+\nu_{A}(x) \leq 1
$$

For each IFS $A$ of $X$,

$$
\pi_{A}(x)=1-\mu_{A}(x)-\nu_{A}(x)
$$

is the intuitionistic fuzzy set index or hesitation margin of $x \in X$. The hesitation margin $\pi_{A}(x)$ is the degree of non-determinacy of $x \in X$, to the set $A$ and $\pi_{A}(x) \in[0,1]$. The hesitation margin is the function that expresses lack of knowledge of whether $x \in X$ or $x \notin X$. Thus,

$$
\mu_{A}(x)+\nu_{A}(x)+\pi_{A}(x)=1 .
$$

We denote the set of all IFSs over $X$ by $I F S(X)$.

Example 2.1. Let $X=\{x, y, z\}$ be a fixed universe of discourse and

$$
A=\left\{\left\langle\frac{0.70,0.10}{x}\right\rangle,\left\langle\frac{0.85,0.05}{y}\right\rangle,\left\langle\frac{0.50,0.20}{z}\right\rangle\right\}
$$

be the intuitionistic fuzzy set in $X$. The hesitation margins of the elements $x, y, z$ to $A$ are respectively,

$$
\pi_{A}(x)=0.20, \pi_{A}(y)=0.10 \text { and } \pi_{A}(z)=0.30 .
$$

Definition 2.3. $[4,6]$ Let $A, B \in I F S(X)$. Then we have the following:

(i) $A^{c}=\left\{\left\langle x, \nu_{A}(x), \mu_{A}(x)\right\rangle \mid x \in X\right\}$.

(ii) $A \cup B=\left\{\left\langle x, \max \left(\mu_{A}(x), \mu_{B}(x)\right), \min \left(\nu_{A}(x), \nu_{B}(x)\right)\right\rangle \mid x \in X\right\}$.

(iii) $A \cap B=\left\{\left\langle x, \min \left(\mu_{A}(x), \mu_{B}(x)\right), \max \left(\nu_{A}(x), \nu_{B}(x)\right)\right\rangle \mid x \in X\right\}$.

(iv) $A \oplus B=\left\{\left\langle x, \mu_{A}(x)+\mu_{B}(x)-\mu_{A}(x) \mu_{B}(x), \nu_{A}(x) \nu_{B}(x)\right\rangle \mid x \in X\right\}$.

(v) $A \otimes B=\left\{\left\langle x, \mu_{A}(x) \mu_{B}(x), \nu_{A}(x)+\nu_{B}(x)-\nu_{A}(x) \nu_{B}(x)\right\rangle \mid x \in X\right\}$. 
Remark 2.1. $[4,6]$ Let $A, B, C \in I F S(X)$. By Definition 2.3, the following properties hold:

$$
\begin{gathered}
\left(A^{c}\right)^{c}=A \\
A \cap A=A \\
A \cup A=A \\
A \oplus A \neq A \\
A \otimes A \neq A \\
A \cap B=B \cap A \\
A \cup B=B \cup A \\
A \oplus B=B \oplus A \\
A \otimes B=B \otimes A
\end{gathered}
$$

$$
\begin{aligned}
& A \cap(B \cap C)=(A \cap B) \cap C \\
& A \cup(B \cup C)=(A \cup B) \cup C \\
& A \oplus(B \oplus C)=(A \oplus B) \oplus C \\
& A \otimes(B \otimes C)=(A \otimes B) \otimes C
\end{aligned}
$$

$$
\begin{aligned}
& A \cap(B \cup C)=(A \cap B) \cup(A \cap C) \\
& A \cup(B \cap C)=(A \cup B) \cap(A \cup C) \\
& A \oplus(B \cup C)=(A \oplus B) \cup(A \oplus C) \\
& A \oplus(B \cap C)=(A \oplus B) \cap(A \oplus C) \\
& A \otimes(B \cup C)=(A \otimes B) \cup(A \otimes C) \\
& A \otimes(B \cap C)=(A \otimes B) \cap(A \otimes C)
\end{aligned}
$$

$$
\begin{aligned}
& (A \cap B)^{c}=A^{c} \cup B^{c} \\
& (A \cup B)^{c}=A^{c} \cap B^{c} \\
& (A \oplus B)^{c}=A^{c} \otimes B^{c} \\
& (A \otimes B)^{c}=A^{c} \oplus B^{c} .
\end{aligned}
$$

Definition 2.4. Let $A \in \operatorname{IFS}(X)$. Then the level/ground set of $A$ is defined by

$$
A_{*}=\left\{x \in X \mid \mu_{A}(x)>0, \nu_{A}(x)<1\right\} .
$$

Certainly, $A_{*}$ is a subset of $X$. 


\section{Concept of intuitionistic fuzzy relations}

The notion of max-min-max composite relation for IFSs has been proposed by Biswas [9] and applied in [14]. Albeit, in this section we introduce an improved composite relation for IFSs.

Definition 3.1. $[9,14]$ Let $X$ and $Y$ be two non-empty sets. An intuitionistic fuzzy relation (IFR), $R$ from $X$ to $Y$ is an IFS of $X \times Y$ characterized by the membership function, $\mu_{R}$ and non-membership function, $\nu_{R}$. An IF relation or IFR from $X$ to $Y$ is denoted by $R(X \rightarrow Y)$.

That is,

$$
R=\left\{\left\langle(x, y), \mu_{R}(x, y), \nu_{R}(x, y)\right\rangle \mid x \in X, y \in Y\right\}
$$

where

$$
\mu_{R}: X \times Y \rightarrow[0,1] \text { and } \nu_{R}: X \times Y \rightarrow[0,1]
$$

satisfy the condition $\mu_{R}(x, y)+\nu_{R}(x, y) \leq 1 \forall(x, y) \in X \times Y$.

\subsection{Intuitionistic fuzzy max-min-max composite relation}

Definition 3.2. $[9,14]$ Let $A \in I F S(X)$. Then, the max-min-max composite relation of

$$
R(X \rightarrow Y)
$$

with $A$ being an IFS, $B$ of $Y$ denoted by $B=R \circ A$, such that its membership and nonmembership functions are defined by

$$
\mu_{B}(y)=\bigvee_{x}\left\{\min \left[\mu_{A}(x), \mu_{R}(x, y)\right]\right\}
$$

and

$$
\nu_{B}(y)=\bigwedge_{x}\left\{\max \left[\nu_{A}(x), \nu_{R}(x, y)\right]\right\}
$$

$\forall x \in X$ and $y \in Y$, where $\bigvee=$ maximum, $\bigwedge=$ minimum.

Definition 3.3. $[9,14]$ Let $Q(X \rightarrow Y)$ and $R(Y \rightarrow Z)$ be two IFRs. Then, the max-min-max composite relation, $R \circ Q$ is an IFR from $X$ to $Z$ such that its membership and non-membership functions are defined by

$$
\mu_{R \circ Q}(x, z)=\bigvee_{y}\left\{\min \left[\mu_{Q}(x, y), \mu_{R}(y, z)\right]\right\}
$$

and

$$
\nu_{R \circ Q}(x, z)=\bigwedge_{y}\left\{\max \left[\nu_{Q}(x, y), \nu_{R}(y, z)\right]\right\}
$$

$\forall(x, z) \in X \times Z$ and $\forall y \in Y$ 
Remark 3.1. [14] From Definitions 3.2 and 3.3, the max-min-max composite relation, $B$ or $R \circ Q$ is calculated by

$$
B=\mu_{B}(y)-\nu_{B}(y) \pi_{B}(y)
$$

$\forall y \in Y$ or

$$
R \circ Q=\mu_{R \circ Q}(x, z)-\nu_{R \circ Q}(x, z) \pi_{R \circ Q}(x, z)
$$

$\forall(x, z) \in X \times Z$.

\subsection{Improved intuitionistic fuzzy composite relation}

In this new composite relation on IFSs, we consider the average of both the membership and non-membership functions, unlike in the case of max-min-max composite relation as discussed above.

Definition 3.4. Let $A \in I F S(X)$. Then, the improved composite relation of

$$
R(X \rightarrow Y)
$$

with $A$ being an IFS, $\mathbf{B}$ of $Y$ denoted by $\mathbf{B}=R \circ A$, such that its membership and nonmembership functions are defined by

$$
\mu_{\mathbf{B}}(y)=\bigvee_{x}\left\{\frac{\mu_{A}(x)+\mu_{R}(x, y)}{2}\right\}
$$

and

$$
\nu_{\mathbf{B}}(y)=\bigwedge_{x}\left\{\frac{\nu_{A}(x)+\nu_{R}(x, y)}{2}\right\}
$$

$\forall x \in X$ and $y \in Y$, where $\bigvee=$ maximum, $\bigwedge=$ minimum.

Definition 3.5. Let $Q(X \rightarrow Y)$ and $R(Y \rightarrow Z)$ be two IFRs. Then, the improved composite relation, $\mathbf{R} \circ \mathbf{Q}$ is an IFR from $X$ to $Z$ such that its membership and non-membership functions are defined by

$$
\mu_{\mathbf{R} \circ \mathbf{Q}}(x, z)=\bigvee_{y}\left\{\frac{\mu_{Q}(x, y)+\mu_{R}(y, z)}{2}\right\}
$$

and

$$
\nu_{\mathbf{R} \circ \mathbf{Q}}(x, z)=\bigwedge_{y}\left\{\frac{\nu_{Q}(x, y)+\nu_{R}(y, z)}{2}\right\}
$$

$\forall(x, z) \in X \times Z$ and $\forall y \in Y$.

Remark 3.2. From Definitions 3.4 and 3.5, the improved composite relation, $\mathbf{B}$ or $\mathbf{R} \circ \mathbf{Q}$ is calculated by

$$
\mathbf{B}=\mu_{\mathbf{B}}(y)-\nu_{\mathbf{B}}(y) \pi_{\mathbf{B}}(y)
$$

$\forall y \in Y$ or

$$
\mathbf{R} \circ \mathbf{Q}=\mu_{\mathbf{R} \circ \mathbf{Q}}(x, z)-\nu_{\mathbf{R} \circ \mathbf{Q}}(x, z) \pi_{\mathbf{R} \circ \mathbf{Q}}(x, z)
$$

$\forall(x, z) \in X \times Z$ 
Proposition 3.1. If $R$ and $S$ are two IFRs on $X \times Y$ and $Y \times Z$, respectively, then

(i) $\left(R^{-1}\right)^{-1}=R$,

(ii) $(S \circ R)^{-1}=R^{-1} \circ S^{-1}$.

\subsection{Numerical verifications}

Before applying this relation to medical diagnosis, we discuss the procedures of the approach step-wisely. Firstly, we use max-min-max composite relation for IFSs and then, the improved composite relation for IFSs. A reliability analysis is conducted to ascertain which of the composite relations provides the best relation by comparing the relational values.

Example 3.1. Let $\tilde{E}, \tilde{F} \in I F S(X)$ for $X=\left\{x_{1}, x_{2}, x_{3}\right\}$. Suppose

$$
\tilde{E}=\left\{\left\langle\frac{0.6,0.2}{x_{1}}\right\rangle,\left\langle\frac{0.4,0.5}{x_{2}}\right\rangle,\left\langle\frac{0.5,0.3}{x_{3}}\right\rangle\right\}
$$

and

$$
\tilde{F}=\left\{\left\langle\frac{0.8,0.1}{x_{1}}\right\rangle,\left\langle\frac{0.7,0.2}{x_{2}}\right\rangle,\left\langle\frac{0.6,0.1}{x_{3}}\right\rangle\right\}
$$

We find the composite relation $B$ by using Definitions 3.2 and 3.3, respectively:

$$
\min \left[\mu_{R}\left(e_{i}, x_{j}\right), \mu_{S}\left(x_{j}, f_{k}\right)\right]=0.6,0.4,0.5
$$

implying that

$$
\mu_{B}\left(e_{i}, f_{k}\right)=\bigvee_{x_{j} \in X}\{0.6,0.4,0.5\}=0.6 .
$$

Clearly, $\min \left[\mu_{R}\left(e_{i}, x_{j}\right), \mu_{S}\left(x_{j}, f_{k}\right)\right]$ is obtained by synthesizing Definitions 3.2 and 3.3. Applying this to $\tilde{E}$ and $\tilde{F}$ as given in the above example, we observe that the minimum value of the membership values of the elements (i.e., $\left.x_{1}, x_{2}, x_{3}\right)$ in $\tilde{E}$ and $\tilde{F}$, respectively, are 0.6, 0.4 and 0.5.

Again,

$$
\max \left[\nu_{R}\left(e_{i}, x_{j}\right), \nu_{S}\left(x_{j}, f_{k}\right)\right]=0.2,0.5,0.3
$$

implying that

$$
\nu_{B}\left(e_{i}, f_{k}\right)=\bigwedge_{x_{j} \in X}\{0.2,0.5,0.3\}=0.2
$$

By explanation, $\max \left[\nu_{R}\left(e_{i}, x_{j}\right), \nu_{S}\left(x_{j}, f_{k}\right)\right]$ is obtained by synthesizing Definitions 3.2 and 3.3. Applying this to $\tilde{E}$ and $\tilde{F}$ as given in the above example, we observe that the maximum value of the non-membership values of the elements (i.e., $\left.x_{1}, x_{2}, x_{3}\right)$ in $\tilde{E}$ and $\tilde{F}$, respectively, are $0.2,0.5$ and 0.3 .

Then

$$
B=0.6-(0.2 \times 0.2)=0.56
$$


Again, finding B using Definitions 3.4 and 3.5 with application to $\tilde{E}$ and $\tilde{F}$, we obtain

$$
\frac{\mu_{R}\left(e_{i}, x_{j}\right)+\mu_{S}\left(x_{j}, f_{k}\right)}{2}=0.7,0.55,0.55
$$

implying that

$$
\mu_{\mathbf{B}}\left(e_{i}, f_{k}\right)=\bigvee_{x_{j} \in X}\{0.7,0.55,0.55\}=0.7
$$

Again,

$$
\frac{\nu_{R}\left(e_{i}, x_{j}\right)+\nu_{S}\left(x_{j}, f_{k}\right)}{2}=0.15,0.35,0.2
$$

implying that

$$
\nu_{\mathbf{B}}\left(e_{i}, f_{k}\right)=\bigwedge_{x_{j} \in X}\{0.15,0.35,0.2\}=0.15
$$

Then

$$
\mathbf{B}=0.7-(0.15 \times 0.15)=0.6775 .
$$

From the aforesaid, the improved composite relation yields a better relation with a greater relational value when compared to max-min-max composite relation.

Now, we consider a case where the IFSs have no equal level sets.

Example 3.2. Let $\tilde{G}, \tilde{H} \in I F S(X)$ for $X=\left\{x_{1}, x_{2}, x_{3}, x_{4}, x_{5}\right\}$. Suppose

$$
\tilde{G}=\left\{\left\langle\frac{0.8,0.1}{x_{1}}\right\rangle,\left\langle\frac{0.5,0.3}{x_{2}}\right\rangle,\left\langle\frac{0.5,0.4}{x_{3}}\right\rangle,\left\langle\frac{0.7,0.2}{x_{5}}\right\rangle\right\}
$$

and

$$
\tilde{H}=\left\{\left\langle\frac{0.7,0.1}{x_{1}}\right\rangle,\left\langle\frac{0.6,0.2}{x_{3}}\right\rangle,\left\langle\frac{0.9,0.1}{x_{4}}\right\rangle\right\} .
$$

Before calculating the composite relations, we rewrite the IFSs as follows:

$$
\tilde{G}=\left\{\left\langle\frac{0.8,0.1}{x_{1}}\right\rangle,\left\langle\frac{0.5,0.3}{x_{2}}\right\rangle,\left\langle\frac{0.5,0.4}{x_{3}}\right\rangle,\left\langle\frac{0.0,1.0}{x_{4}}\right\rangle,\left\langle\frac{0.7,0.2}{x_{5}}\right\rangle\right\}
$$

and

$$
\tilde{H}=\left\{\left\langle\frac{0.7,0.1}{x_{1}}\right\rangle,\left\langle\frac{0.0,1.0}{x_{2}}\right\rangle,\left\langle\frac{0.6,0.2}{x_{3}}\right\rangle,\left\langle\frac{0.9,0.1}{x_{4}}\right\rangle,\left\langle\frac{0.0,1.0}{x_{5}}\right\rangle\right\} .
$$

Now we find $B$ using Definitions 3.2 and 3.3, respectively, as follows:

$$
\min \left[\mu_{R}\left(g_{i}, x_{j}\right), \mu_{S}\left(x_{j}, h_{k}\right)\right]=0.7,0.0,0.5,0.0,0.0
$$

implying that

$$
\mu_{B}\left(g_{i}, h_{k}\right)=\bigvee_{x_{j} \in X}\{0.7,0.0,0.5,0.0,0.0\}=0.7
$$


Again,

$$
\max \left[\nu_{R}\left(g_{i}, x_{j}\right), \nu_{S}\left(x_{j}, h_{k}\right)\right]=0.1,1.0,0.4,1.0,1.0,
$$

implying that

$$
\nu_{B}\left(g_{i}, h_{k}\right)=\bigwedge_{x_{j} \in X}\{0.1,1.0,0.4,1.0,1.0\}=0.1 .
$$

Thus

$$
B=0.7-(0.1 \times 0.2)=0.68 .
$$

Also, finding B using Definitions 3.4 and 3.5, we obtain

$$
\frac{\mu_{R}\left(g_{i}, x_{j}\right)+\mu_{S}\left(x_{j}, h_{k}\right)}{2}=0.75,0.25,0.55,0.45,0.35
$$

implying that

$$
\mu_{\mathbf{B}}\left(g_{i}, h_{k}\right)=\bigvee_{x_{j} \in X}\{0.75,0.25,0.55,0.45,0.35\}=0.75
$$

Again,

$$
\frac{\nu_{R}\left(g_{i}, x_{j}\right)+\nu_{S}\left(x_{j}, h_{k}\right)}{2}=0.1,0.65,0.3,0.55,0.6,
$$

implying that

$$
\nu_{\mathbf{B}}\left(g_{i}, h_{k}\right)=\bigwedge_{x_{j} \in X}\{0.1,0.65,0.3,0.55,0.6\}=0.1 .
$$

Then

$$
\mathbf{B}=0.75-(0.1 \times 0.15)=0.735 .
$$

In this case also, the improved composite relation gives a better relation compared to max-minmax composite relation.

Table 1 gives the comparative analysis of the improved composite relation $\mathbf{B}$ and max-minmax composite relation $B$ for IFSs. In what follows, the relational value of $\mathbf{B}$ is greater than that of $B$. This shows that $\mathbf{B}$ provides better intuitionistic fuzzy relation when compared to $B$.

\begin{tabular}{|l|l|l|}
\hline IFR & Example 3.1 & Example 3.2 \\
\hline$B$ & 0.5600 & 0.6800 \\
B & 0.6775 & 0.7350 \\
\hline
\end{tabular}

Table 1. Comparative analysis 


\section{Improved composite relation for intuitionistic fuzzy sets in medical diagnosis}

In this section, we present an application of IFSs to medical diagnosis using both the max-minmax composite relation and the improved composite relation for IFSs. In a given pathology, suppose $S$ is a set of symptoms, $D$ is a set of diseases, and $P$ is a set of patients. We define intuitionistic fuzzy medical knowledge as an intuitionistic fuzzy relation $R$ from the set of symptoms $S$ to the set of diseases $D$ (i.e., on $S \times D$ ), which reveals the degree of association and the degree of non-association between symptoms and diseases.

In [14], the notion of intuitionistic fuzzy medical diagnosis was discussed via the following methodology;

(i) determination of symptoms,

(ii) formulation of medical knowledge based on intuitionistic fuzzy relations, and

(iii) determination of diagnosis on the basis of composition of intuitionistic fuzzy relations.

Let $A$ be an IFS of the set $S$, and $R$ be an IFR from $S$ to $D$. Then, max-min-max composite relation $B$ for IFS $A$ with the IFR $R(S \rightarrow D)$ denoted by

$$
B=A \circ R
$$

signifies the state of the patient in terms of diagnosis as an IFS $B$ of $D$ with the membership function given by

$$
\mu_{B}(d)=\bigvee_{s \in S}\left\{\min \left[\mu_{A}(s), \mu_{R}(s, d)\right]\right\}
$$

and the non-membership function given by

$$
\nu_{B}(d)=\bigwedge_{s \in S}\left\{\max \left[\nu_{A}(s), \nu_{R}(s, d)\right]\right\}
$$

$\forall d \in D$

Similarly, the improved composite relation B for IFS $A$ with the IFR $R(S \rightarrow D)$ denoted by

$$
\mathbf{B}=A \circ R
$$

signifies the state of the patient in terms of diagnosis as an IFS $\mathbf{B}$ of $D$ with the membership function given by

$$
\mu_{\mathbf{B}}(d)=\bigvee_{s \in S}\left\{\frac{\mu_{A}(s)+\mu_{R}(s, d)}{2}\right\}
$$

and the non-membership function given by

$$
\nu_{\mathbf{B}}(d)=\bigwedge_{s \in S}\left\{\frac{\nu_{A}(s)+\nu_{R}(s, d)}{2}\right\}
$$

$\forall d \in D$ 
If the state of a given patient $P$ is described in terms of an IFS $A$ of $S$, then $P$ is assumed to be assigned a diagnosis in terms of IFRs $B$ and $\mathbf{B}$ of $D$, through an IFR $R$ of intuitionistic medical knowledge from $S$ to $D$ which is assumed to be given by a doctor who is able to translate his/her own observation of the fuzziness involved in degrees of association and non-association, respectively, between symptoms and diagnosis.

Now, we extend these concepts to a finite number of patients. Let there be $n$ patients $p_{i}$ for $i=1,2, \ldots, n$ in a given laboratory. Thus $p_{i} \in P$ (or simply, $p \in P$ ). Let $R$ be an IFR $(S \rightarrow D$ ) and construct an IFR $Q$ from the set of patients $P$ to the set of symptoms $S$. Clearly, the maxmin-max composite relation $B$ of IFRs $R$ and $Q(B=R \circ Q)$ designates the state of patients $p$ in terms of the diagnosis as an IFR from $P$ to $D$ given by the membership function

$$
\mu_{B}(p, d)=\bigvee_{s \in S}\left\{\min \left[\mu_{Q}(p, s), \mu_{R}(s, d)\right]\right\}
$$

and the non-membership function given by

$$
\nu_{B}(p, d)=\bigwedge_{s \in S}\left\{\max \left[\nu_{Q}(p, s), \nu_{R}(s, d)\right]\right\}
$$

$\forall p \in P$ and $\forall d \in D$.

Similarly, the improved composite relation $\mathbf{B}$ of IFRs $R$ and $Q(\mathbf{B}=R \circ Q)$ designates the state of patients $p$ in terms of the diagnosis as an IFR from $P$ to $D$ given by the membership function

$$
\mu_{\mathbf{B}}(p, d)=\bigvee_{s \in S}\left\{\frac{\mu_{Q}(p, s)+\mu_{R}(s, d)}{2}\right\}
$$

and the non-membership function given by

$$
\nu_{\mathbf{B}}(p, d)=\bigwedge_{s \in S}\left\{\frac{\nu_{Q}(p, s)+\nu_{R}(s, d)}{2}\right\}
$$

$\forall p \in P$ and $\forall d \in D$.

For a given $R$ and $Q$, the relation $B=R \circ Q$ or $\mathbf{B}=R \circ Q$ can be computed. From the knowledge of $Q$ and $R$, one may find $B$ and $\mathbf{B}$ of the IFR for which

$$
B=\mu_{B}(p, d)-\nu_{B}(p, d) \pi_{B}(p, d)
$$

and

$$
\mathbf{B}=\mu_{\mathbf{B}}(p, d)-\nu_{\mathbf{B}}(p, d) \pi_{\mathbf{B}}(p, d)
$$

are the greatest.

Obviously, $R$ is a significant IFR translating the higher degrees of association and lower degrees of non-association of symptoms as well as degrees of hesitation to the diseases, an approach to intuitionistic fuzzy medical knowledge. From this approach, one may infer diagnosis from symptoms in the sense of a paired value, one being the degree of association and other the degree of non-association. 


\subsection{Case study}

Suppose four patients viz; Lil, Jones, Deby, and Inas visit a given laboratory for medical diagnosis. They are observed to have the following symptoms; temperature, headache, stomach pain, cough, and chest pain. That is, the set of patients $P$ is

$$
P=\{\text { Lil, Jones, Deby, Inas }\},
$$

and the set of symptoms $S$ is

$$
S=\{\text { temperature, headache, stomach pain, cough, chest pain }\}
$$

The intuitionistic fuzzy relation $Q(P \rightarrow S)$ is given hypothetically in Table 2 .

Let the set of diseases $D$ the patients are suspected to be suffering from be

$$
D=\{\text { viral fever, malaria, typhoid, stomach problem, heart problem }\}
$$

The intuitionistic fuzzy relation $R(S \rightarrow D)$ is given hypothetically in Table 3 . Note that, the data in Tables 2 and 3 are extracted from [14]. The values of the membership and non-membership functions of the composite relations $B=R \circ Q$ is given in Table 4 using Equations 1 and 2, and $\mathbf{B}=R \circ Q$ is given in Table 5 using Equations 3 and 4. After finding the degree of hesitation $(\pi=1-[\mu+\nu])$, we calculate $B$ and $\mathbf{B}$ as given in Tables 6 and 7 using Equations 5 and 6, respectively.

\begin{tabular}{|l|l|l|l|l|l|}
\hline$Q$ & temperature & headache & stomach pain & cough & chest pain \\
\hline Lil & $\langle 0.8,0.1\rangle$ & $\langle 0.6,0.1\rangle$ & $\langle 0.2,0.8\rangle$ & $\langle 0.6,0.1\rangle$ & $\langle 0.1,0.6\rangle$ \\
Jones & $\langle 0.0,0.8\rangle$ & $\langle 0.4,0.4\rangle$ & $\langle 0.6,0.1\rangle$ & $\langle 0.1,0.7\rangle$ & $\langle 0.1,0.8\rangle$ \\
Deby & $\langle 0.8,0.1\rangle$ & $\langle 0.8,0.1\rangle$ & $\langle 0.0,0.6\rangle$ & $\langle 0.2,0.7\rangle$ & $\langle 0.0,0.5\rangle$ \\
Inas & $\langle 0.6,0.1\rangle$ & $\langle 0.5,0.4\rangle$ & $\langle 0.3,0.4\rangle$ & $\langle 0.7,0.2\rangle$ & $\langle 0.3,0.4\rangle$ \\
\hline
\end{tabular}

Table 2. $Q(P \rightarrow S)$

\begin{tabular}{|l|l|l|l|l|l|}
\hline$R$ & viral fever & malaria fever & typhoid fever & stomach problem & chest problem \\
\hline temperature & $\langle 0.4,0.0\rangle$ & $\langle 0.7,0.0\rangle$ & $\langle 0.3,0.3\rangle$ & $\langle 0.1,0.7\rangle$ & $\langle 0.1,0.8\rangle$ \\
headache & $\langle 0.3,0.5\rangle$ & $\langle 0.2,0.6\rangle$ & $\langle 0.6,0.1\rangle$ & $\langle 0.2,0.4\rangle$ & $\langle 0.0,0.8\rangle$ \\
stomach & $\langle 0.1,0.7\rangle$ & $\langle 0.0,0.9\rangle$ & $\langle 0.2,0.7\rangle$ & $\langle 0.8,0.0\rangle$ & $\langle 0.2,0.8\rangle$ \\
pain & & & & & \\
cough & $\langle 0.4,0.3\rangle$ & $\langle 0.7,0.0\rangle$ & $\langle 0.2,0.6\rangle$ & $\langle 0.2,0.7\rangle$ & $\langle 0.2,0.8\rangle$ \\
chest pain & $\langle 0.1,0.7\rangle$ & $\langle 0.1,0.8\rangle$ & $\langle 0.1,0.9\rangle$ & $\langle 0.2,0.7\rangle$ & $\langle 0.8,0.1\rangle$ \\
\hline
\end{tabular}

Table 3. $R(S \rightarrow D)$ 


\begin{tabular}{|l|l|l|l|l|l|}
\hline$\mu_{B}, \nu_{B}$ & viral fever & malaria fever & typhoid fever & stomach problem & chest problem \\
\hline Lil & $\langle 0.40,0.10\rangle$ & $\langle 0.70,0.10\rangle$ & $\langle 0.60,0.10\rangle$ & $\langle 0.20,0.40\rangle$ & $\langle 0.20,0.60\rangle$ \\
Jones & $\langle 0.30,0.50\rangle$ & $\langle 0.20,0.60\rangle$ & $\langle 0.40,0.40\rangle$ & $\langle 0.60,0.10\rangle$ & $\langle 0.10,0.70\rangle$ \\
Deby & $\langle 0.40,0.10\rangle$ & $\langle 0.70,0.10\rangle$ & $\langle 0.60,0.10\rangle$ & $\langle 0.20,0.40\rangle$ & $\langle 0.20,0.50\rangle$ \\
Inas & $\langle 0.40,0.10\rangle$ & $\langle 0.70,0.10\rangle$ & $\langle 0.50,0.30\rangle$ & $\langle 0.30,0.40\rangle$ & $\langle 0.30,0.40\rangle$ \\
\hline
\end{tabular}

Table 4. $\mu_{B}(p, d)$ and $\nu_{B}(p, d)$

\begin{tabular}{|l|l|l|l|l|l|}
\hline$\mu_{\mathbf{B}}, \nu_{\mathbf{B}}$ & viral fever & malaria fever & typhoid fever & stomach problem & chest problem \\
\hline Lil & $\langle 0.60,0.05\rangle$ & $\langle 0.75,0.05\rangle$ & $\langle 0.60,0.10\rangle$ & $\langle 0.50,0.25\rangle$ & $\langle 0.45,0.35\rangle$ \\
Jones & $\langle 0.60,0.05\rangle$ & $\langle 0.40,0.35\rangle$ & $\langle 0.50,0.25\rangle$ & $\langle 0.70,0.05\rangle$ & $\langle 0.45,0.45\rangle$ \\
Deby & $\langle 0.60,0.05\rangle$ & $\langle 0.75,0.05\rangle$ & $\langle 0.70,0.10\rangle$ & $\langle 0.50,0.25\rangle$ & $\langle 0.45,0.30\rangle$ \\
Inas & $\langle 0.55,0.05\rangle$ & $\langle 0.70,0.05\rangle$ & $\langle 0.55,0.20\rangle$ & $\langle 0.55,0.20\rangle$ & $\langle 0.55,0.25\rangle$ \\
\hline
\end{tabular}

Table 5. $\mu_{\mathbf{B}}(p, d)$ and $\nu_{\mathbf{B}}(p, d)$

\begin{tabular}{|l|l|l|l|l|l|}
\hline$B$ & viral fever & malaria fever & typhoid fever & stomach problem & chest problem \\
\hline Lil & 0.35 & 0.68 & 0.57 & 0.04 & 0.08 \\
Jones & 0.20 & 0.08 & 0.32 & 0.57 & 0.04 \\
Deby & 0.35 & 0.68 & 0.57 & 0.04 & 0.05 \\
Inas & 0.32 & 0.68 & 0.44 & 0.18 & 0.18 \\
\hline
\end{tabular}

Table 6. $B=\mu_{B}-\nu_{B} \pi_{B}$

\begin{tabular}{|l|l|l|l|l|l|}
\hline B & viral fever & malaria fever & typhoid fever & stomach problem & chest problem \\
\hline Lil & 0.5825 & 0.7400 & 0.5700 & 0.4375 & 0.3800 \\
Jones & 0.5825 & 0.3125 & 0.4375 & 0.6875 & 0.4050 \\
Deby & 0.5825 & 0.7400 & 0.6800 & 0.4375 & 0.3750 \\
Inas & 0.5300 & 0.6875 & 0.5000 & 0.5000 & 0.5000 \\
\hline
\end{tabular}

Table 7. $\mathbf{B}=\mu_{\mathbf{B}}-\nu_{\mathbf{B}} \pi_{\mathbf{B}}$

\subsection{Decisions on the patients' medical conditions}

With the aid of Tables 6 and 7, we present the decision-making. Decisions are made based on the greatest value of relation between patients and diseases. From Table 6, we notice that: Lil is suffering from malaria fever; Jones is suffering from stomach problem; Deby is suffering from malaria fever; Inas is suffering from malaria fever.

Similarly, we notice from Table 7 that: Lil is suffering from malaria fever; Jones is suffering from stomach problem; Deby is suffering from malaria fever; Inas is suffering from malaria fever.

Both decisions from Tables 6 and 7 coincide but with a difference that the improved composite relation for IFSs gives higher relational values. This shows that, the improved intuitionistic fuzzy composite relation yields a better result when compared to max-min-max composite relation for IFSs applied in [14]. 


\section{Conclusion}

IFS theory is a resourceful mathematical framework in the fuzzy family with a higher ability to tackle uncertainty embedded in decision-making. A number of applications of IFSs have been discussed in literature. In this paper, the De et al.'s approach for medical diagnostic process was considered and improved upon. We showed that the improved intuitionistic fuzzy composite relation yields a better output when compared to the De et al.'s approach. Thus, the need for its application to solving medical diagnosis problem and other related problems cannot be overemphasized. The improved composite relation proposed in this paper could be used as a viable tool in applying IFSs to multi-criteria decision-making problems. Albeit, it is suggested to consider this approach from object-oriented perspective for quick output in further research.

\section{Acknowledgements}

The authors are thankful to the Editor-in-Chief Professor K. T. Atanassov for his technical comments and to the anonymous reviewers for their suggestions which have improved the quality of this paper.

\section{References}

[1] Atanassov K. T. (1983). Intuitionistic Fuzzy Sets, VII ITKR Session, Sofia, 20-23 June 1983 (Deposed in Centr. Sci.-Techn. Library of the Bulg. Acad. of Sci., 1697/84) (in Bulgarian). Reprinted: Int. J. Bioautomation, 2016, 20(S1), S1-S6.

[2] Atanassov, K. T. (1986). Intuitionistic fuzzy sets, Fuzzy Sets and Systems, 20 (1), 87-96.

[3] Atanassov, K. T. (1989). More on intuitionistic fuzzy sets, Fuzzy Sets and Systems, 33, 3745.

[4] Atanassov, K. T. (1994). New operations defined over intuitionistic fuzzy sets, Fuzzy Sets and Systems, 61(2), 137-142.

[5] Atanassov, K. T. (1999). Intuitionistic Fuzzy Sets: Theory and Applications, Physica-Verlag, Heidelberg.

[6] Atanassov, K. T. (2012). On Intuitionistic Fuzzy Sets Theory, Springer, Berlin.

[7] Atanassov, K. T. \& Gargov, G. (1990). Intuitionistic fuzzy logic, C. R. Acad. Bulgare Sc., 43 (3), 9-12.

[8] Atanassov, K. T. \& Georgiev, C. (1993). Intuitionistic fuzzy Prolog, Fuzzy Sets and Systems, $53,121-128$. 
[9] Biswas, R. (1997). Intuitionistic fuzzy relations, Bull. Sous. Ens. Flous. Appl. (BUSEFAL), 70, 22-29.

[10] Burillo, P. \& Bustince, H. (1995). Intuitionistic fuzzy relations (Part 1), Mathware Soft Comput., 2, 5-38.

[11] Burillo, P. \& Bustince, H. (1996). Structures on intuitionistic fuzzy relations, Fuzzy Sets and Systems, 78, 293-303.

[12] Davvaz, B. \& Sadrabadi, E. H. (2016). An application of intuitionistic fuzzy sets in medicine, Int. J. Biomath., 9 (3), 1650037.

[13] De, S. K., Biswas, R. \& Roy, A. R. (2000). Some operations on intuitionistic fuzzy sets, Fuzzy Sets and Systems, 114, 477-484.

[14] De, S. K., Biswas, R. \& Roy, A. R. (2001). An application of intuitionistic fuzzy sets in medical diagnosis, Fuzzy Sets and Systems, 117 (2), 209-213.

[15] De Baets, B. \& Etienne, E. K. (1993). Fuzzy relational compositions, Fuzzy Sets and Systems, 60, 109-120.

[16] De Baets, B. \& Etienne, E. K. (1994). Fuzzy relations and applications, Adv. Electron. Electron. Phys., 89, 255-324.

[17] Deschrijver, G. \& Etienne, E. K. (2003). On the composition of intuitionistic fuzzy relations, Fuzzy Sets and Systems, 136, 333-361.

[18] Ejegwa, P. A. (2015). Intuitionistic fuzzy sets approach in appointment of positions in an organization via max-min-max rule, Global J. Sci. Frontier Research: F Math. Decision Sci., 15 (6), 1-6.

[19] Ejegwa, P. A. \& Modom, E. S. (2015). Diagnosis of viral hepatitis using new distance measure of intuitionistic fuzzy sets, Intern. J. Fuzzy Mathematical Archive, 8 (1), 1-7.

[20] Ejegwa, P. A., Akubo, A. J. \& Joshua, O. M. (2014). Intuitionistic fuzzzy sets in career determination, J. Info. Computing Sci., 9 (4), 285-288.

[21] Ejegwa, P. A., Onoja, A. M. \& Emmanuel, I. T. (2014). A note on some models of intuitionistic fuzzy sets in real life situations, J. Global Research Math. Arch., 2 (5), 42-50.

[22] Ejegwa, P. A., Onoja, A. M. \& Chukwukelu, S. N. (2014). Application of intuitionistic fuzzy sets in research questionnaire, J. Global Research Math. Arch., 2 (5), 51-54.

[23] Ejegwa, P. A., Tyoakaa, G. U. \& Ayenge, A. M. (2016). Application of intuitionistic fuzzy sets in electoral system, Intern. J. Fuzzy Mathematical Archive, 10 (1), 35-41. 
[24] Sanchez, E. (1976). Resolution of composition fuzzy relation equations, Information and Control, 30, 38-48.

[25] Szmidt, E. \& Kacprzyk, J. (2001). Intuitionistic fuzzy sets in some medical applications, Notes on Intuitionistic Fuzzy Sets, 7 (4), 58-64.

[26] Szmidt, E. \& Kacprzyk, J. (2002). An intuitionistic fuzzy set based approach to intelligent data analysis: an application to medical diagnosis. Recent Advances in Intelligent Paradigms and Applications, Springer, Berlin, 57-70.

[27] Szmidt, E. \& Kacprzyk, J. (2004). Medical diagnostic reasoning using a similarity measure for intuitionistic fuzzy sets, Notes on Intuitionistic Fuzzy Sets, 10 (4), 61-69.

[28] Szmidt, E. \& Kacprzyk, J. (2005). A similarity measure for intuitionistic fuzzy sets and its application in supporting medical diagnostic reasoning. Lecture Notes in Artificial Intelligence, Vol. 3070, Springer, Berlin, 388-393.

[29] Todorova, L., Atanassov, K. T., Hadjitodorov, S. \& Vassilev, P. (2007). On an intuitionistic fuzzy approach for decision-making in medicine (Part 1), Int. J. Bioautomation, 6, 92-101.

[30] Todorova, L., Atanassov, K. T., Hadjitodorov, S. \& Vassilev, P. (2007). On an intuitionistic fuzzy approach for decision-making in medicine (Part 2), Int. J. Bioautomation, 7, 64-69.

[31] Zadeh, L. A. (1965). Fuzzy sets, Information and Control, 8, 338-353 